\title{
AutocarreqMereq: A Tool to Elicit Trust Requirements of Autonomous Car
}

\author{
Massila Kamalrudin, Halimaton Hakimi, Suriati Akmal
}

\begin{abstract}
Automakers have become more interested in developing autonomous cars. The acceptance rate of autonomous vehicles by customers is, however, limited. In view of the confidence that consumers embrace the autonomous cars is one of the key determinants. This paper therefore introduces Autocarreq. Mereq, an automated tool to generate trust requirements for self-sufficient cars. This automated tool uses the TReAC library to support capturing and checking accuracy occurring in autonomous car requirements. Autacarreq.Mereq tool which runs in table and mobile support, is compatible to minimise time for on-site capture and elicitation of trust requirements. This paper describes the Autocarreq.Mereq approach and demonstrates its use to capture and elicited trust requirements of autonomous car.
\end{abstract}

Keywords: Trust Requirements, Elicitation Requirements, Autonomous Car Requirements, TReAC Library

\section{INTRODUCTION}

In automotive technology, especially in autonomous car growth referred to as driverless cars, the automotive industry has experienced a new revolution. The Mega Science 3.0 roadmap 2020-2050. The sector needs to deliver safer and more effective driver-free driving. Autonomous cars (ACs) are vehicles that do not have direct driver input and that include the control function. The National Traffic Safety Administration for Highways (NHTSA, 2016) defines the safety-critical control function in autonomous cars, such as steering, brake assistance, automatic parking and other features without a driver input. [1]. Although driving ACs have been argued for a considerable improvement of safety through reduction of accidents due to errors in human beings and human distraction[2], the amount of adoption by the user of autonomous cars remains minimal. In this situation, trust criteria should be linked to user trust in the independent vehicle, which arises from the attempts of the provider in considering the security, security, confidentiality and efficiency that satisfy the user's needs. This is because the precise definition of trust criteria helps consumers select and use a high amount of security AC and prevent customers from involving in accidents. This research applies layers of anthropomorphic signals to an artificial driver in order to address this problem from a psychological point of view and explains the process in which such signals encourage a

Revised Version Manuscript Received on September 16, 2019.

Massila Kamalrudin, Associate Professor Doctor, Faculty of Information and Communication technology, Universiti Teknikal Malaysia Melaka, Melaka, Malaysia

(Email: massila@utem.edu.my)

Halimaton Hakimi, Faculty of Information and Communication technology, Universiti Teknikal Malaysia Melaka, Melaka, Malaysia

Suriati Akmal, Doctor, Faculty of Manufacturing, Universiti Teknikal Malaysia Melaka, Melaka, Malaysia
(Email: : halimaton.mpp1516@gmail.com)

favorable evaluation and perception of unmanaged driving systems.

Moreover, a lack of trust is one of the consequences of reducing acceptance by the user in ACs. Acceptance is a concept after exposure and usually measured after exposure, which allows users to express their support via attitude or behaviour. Most users can not accept a vehicle which, if required, is not manually controlled by humans. This shows clearly that user acceptance is the primary obstacle for user adoption of auto-contained or total automation technology [2]. In fact, everyone wants to safeguard their own car at all costs. This aspiration is in line with the growth of autonomous cars that address "safety dilemmas" in order to reduce the amount of deaths. The development of the autonomous car is anticipated to end up reducing the collision rates, leading to an enhancement in safety. In this situation, autonomous car design must take into account trust and user acceptance problems. Therefore, we create an Autocarreq. Mereq tool to address all issues and problems in the autonomous car development process.

This paper describes the development of tool support which is able to automatically capture trust requirements by matching keywords in comparing information from textual requirements in TReAC library. We begin by descripting the process of trust requirements approach. Next, we present the development TReAC Library. Then, we explain the usage example of Autocarreq.Mereq tool by using persona. After that, we discuss on the result of user perception on the usability. Finally, we discuss implication of this study and prototype as well as our future work.

\section{MOTIVATION AND RELATED WORKS}

There is a work from [3], who have developed the Malay-English Requirements Engineering Tool (MEReq) (Kamalrudin et al. 2014), A tool for developing and checking requirements consistencies. MEReq used models from the interaction pattern libraries with the same context as the domain library, the Essential Using Case (EUC) and the Essential User Interface (EUI). While MEReq fulfills multi-lingual criteria for multiple company application fields in both Malaysian and English, this language requirement does not apply to the autonomous car domain. In particular, MEReq has the ability to validate the captured requirements when the requirements are identified in inconsistency to essential requirements stored in the pattern library. We think that MEReq is beneficial for accurate requirements and it 
An method called Athena is suggested by[ 4]. Athena is a collaborative approach based on the group storytelling approach to eliciting demands. In this strategy, stakeholders tell stories about systems that promote certain activities, both past and present. It defines a solution to issues such as point of view, mental model and the expectation of user and analyst difference. Athena is also a collective knowledge strategy that progressively develops the system requirements from a user narrative to the definition of usesThe stories are then combined into one story. The stories are then transformed into scenarios and into Use cases from the scenarios. The solution is a knowledge model based on system reports, a collective building technique and an interaction support tool. Although the method was applied to eliciting demands, this method still has to be applied in trust requirements, in particular in the car industry.

In order to enhance the present approach to eliciting demands using the paper prototype, [5] also suggested a fresh strategy. This paper prototype represents the overall design of the system. This can be viewed by drawing hands or by means of the graphics program. Based upon user feedback and collected requirements, this strategy is modifiable. However, the specifications are stabilized once the prototypes have been completed. The prototype may be removed once the specifications have been specified after the stabilization process. A postgraduate team has carried out three case studies that will assist them to analyze the utility of this method. The results of the research show that this strategy is appropriate for small and medium-sized projects. Although this approach allows for simple comprehension of the stakeholder's demands, it is not appropriate in the autonomous field. This is because the autonomous domain in comparison with other domains is a relatively large and complex domain. The autonomous domain also covers human life that require accurate explanation of demands so that the terms used are not misunderstood.

There are various tools for eliciting requirements, however tools for eliciting trust requirements of autonomous car is almost non-existence. This finding indicates that there is very limited work in the autonomous car domain.

\section{TRUST REQUIREMENTS APPROACH}

We have developed a trust requirements approach that supports the analysis process of trust requirements for developing acceptable autonomous car. This prototype tool is an extension of the earlier tool that runs in both mobile and web application. These tools help to assist automotive engineer to analyze the trust requirements or trustworthy level from client-stakeholder requirements. Therefore, automotive engineer can use the AutocarreqsMereq tool to analyze the trust requirements of autonomous car. Figure 1 shows an overview of our trust requirements approach. The process starts with automotive engineer sit together with their client to elicit the requirements of autonomous car. Table 1 shows a process of trust requirements approach.

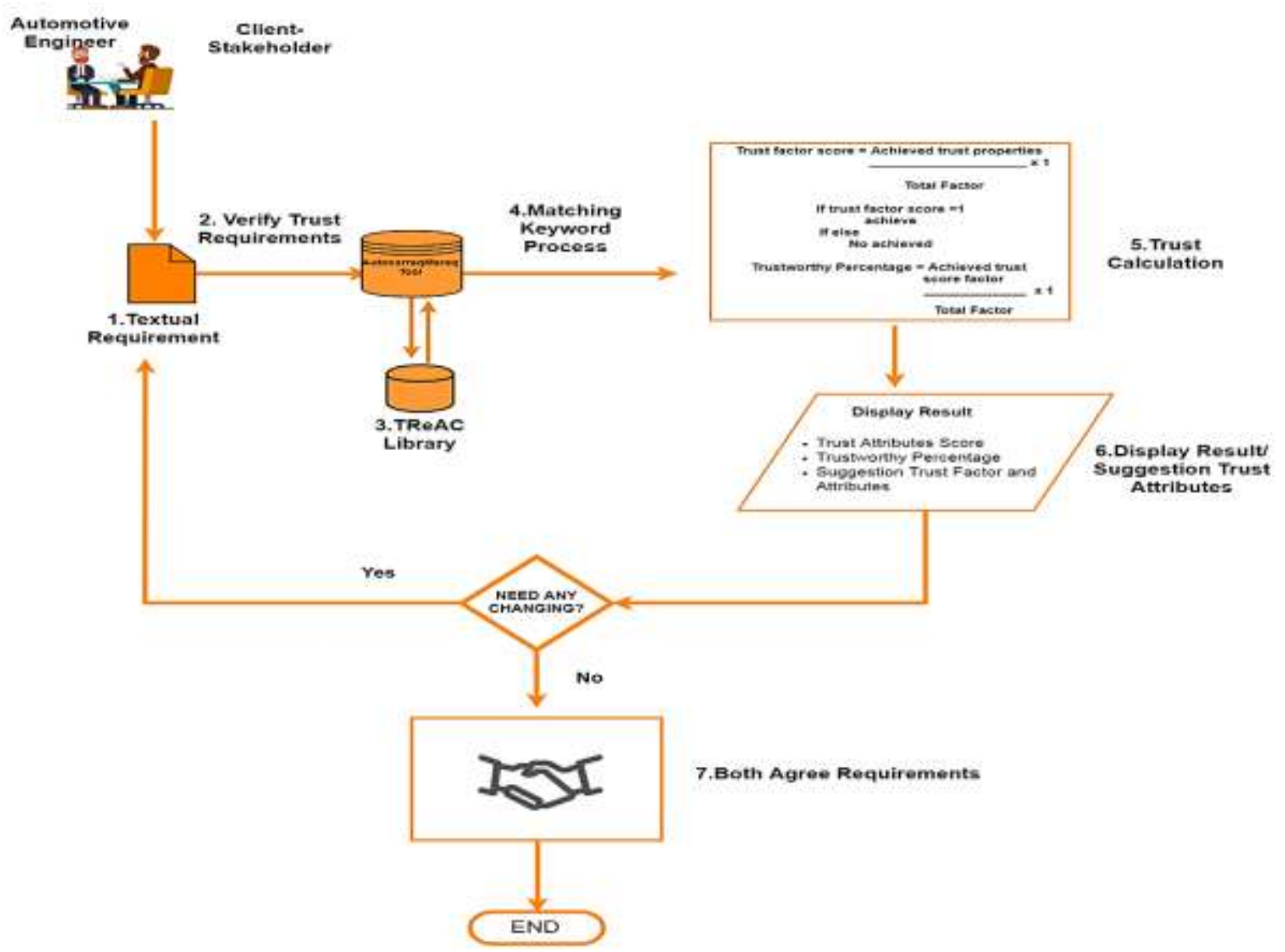

Figure 1: Trust Requirements Approach 
Table 1: The Process of Trust Requirements Approach

Process (1): Requirement return by stakeholders with automotive engineer sit together to elicit the requirements of autonomous car. This requirements return in textual language.

Process (2): The requirements inserted into Autocarreq.Mereq tool to verify trust requirements.

Process (3): Here actually Autocarreq.Mereq process by TReAC Library. Where TReAC Library used to analyses the requirements especially in trust properties and trust attributes.

Process (4): Next, Autocarreq.Mereq also check level of trust by calculating the trust level using the keywords matching process by comparing the information in TReAC Library and from the textual requirements.

Process (5): Then, trust requirement calculated trust level. The propose rule to calculate trustworthy percentage of trust requirements as below in Figure 2.

$$
\begin{gathered}
\begin{array}{c}
\text { Trust Factor Score }=\frac{\text { Achived trust properties }}{\text { Total factor }} \times 1 \\
\text { If trust factor score }=1 \\
\text { Achieved }
\end{array} \\
\text { If else } \begin{array}{c}
\text { Not achieved } \\
\text { Trustworthy Percentage }=\frac{\text { Achieved trust factor }}{\text { Total Factor }} \times 100
\end{array}
\end{gathered}
$$

Figure 2: Rule to calculate trustworthy percentage of trust requirements

Figure 2 show that mathematical formula to calculate level of trustworthy percentage. Before measure the trustworthy percentage, there is a need to calculate trust factor score. This trust factor score calculation is based on keywords of trust properties. If trust factor score $=1$, that means it has achieved trust factor score. If trust factor score $<1$, it means that it did not achieve the trust factor score. Trustworthy percentage calculates based on achieved trust factor. Therefore trust factor must be achieved and trustworthy percentage can be calculated. Otherwise, there is zero percentage of trustworthy. This rule functioning in TReAC Library is to help automotive engineer to calculate the percentage of trust requirements automatically and accuracy results.

Process (6): After calculation, the results will be displayed on the three aspects, namely: trust score, trustworthy percentage and suggestion on the trust factor.

Process (7): Results obtained from the system will propose or suggest changes to the trust attributes to the client-stakeholders if any or necessary.

Process (8): If there no changes, this will end with agreed requirements.

\subsection{Trust Requirements Autonomous Car Pattern Library (TReAC Library)}

We have developed a trust requirement autonomous car (TReAC) pattern library to support and analyze the trust related attributed and properties from the related trust requirements. This $\mathrm{TReAC}$ pattern library is derived and adapted from TReAC model [6]. The purpose of developing TReAC Library is to store all the trust attributes and properties. The proposed TReAc Library is to help automotive engineer to analyze correctly all trust requirements before proceeding to the development of autonomous car. The usefulness of trust requirements autonomous car pattern library is generic and can be utilized and applied for any other domain of application. Furthermore, the usage of TReAC library can minimize human time effort to analyze the trust requirements. The trust requirements autonomous car (TReAC) pattern library is based on the associated trust attributed and trust properties as explained in previous chapter. The relationship between trust attributes and trust properties illustrates in Figure 3. The relationship between trust-assigned property and trust property is based on one or more relationships (1:M). The examples for trust properties relating to trust properties are shown in Table 2.

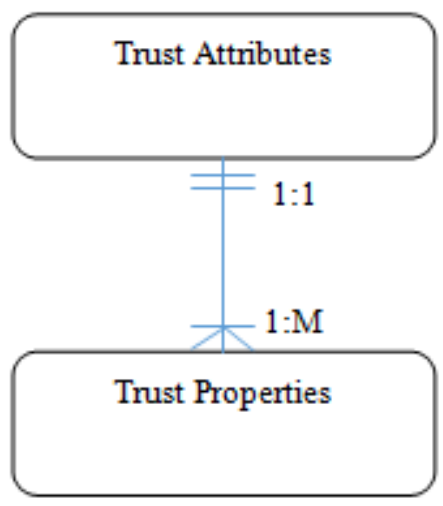

Figure 3: The relationship between trust attributes and trust properties 
Table 2: The examples of trust properties that are related to trust properties.

\begin{tabular}{|c|c|c|}
\hline $\begin{array}{l}\text { Type } \\
\text { Requirement }\end{array}$ & $\begin{array}{l}\text { Trust } \\
\text { Attribute (Ta) }\end{array}$ & Trust Properties (Tp) \\
\hline \multirow{10}{*}{$\begin{array}{l}\text { Trust } \\
\text { Requirements }\end{array}$} & \multirow{5}{*}{ Safety } & Automatic steering \\
\hline & & $\begin{array}{l}\text { Intelligent parking assist } \\
\text { system }\end{array}$ \\
\hline & & Blind spot system \\
\hline & & Adaptive light control \\
\hline & & $\begin{array}{ll}\text { Camera } & \text { sensor } \\
\text { technology } & \end{array}$ \\
\hline & \multirow[t]{5}{*}{ Security } & Anti-Theft System \\
\hline & & Vehicle Access System \\
\hline & & Data sharing \\
\hline & & $\begin{array}{l}\text { Global positioning } \\
\text { system (GPS) }\end{array}$ \\
\hline & & $\begin{array}{lr}\text { Visible } & \text { Vehicle } \\
\text { Identification } & \text { Number } \\
\text { (VIN) } & \end{array}$ \\
\hline
\end{tabular}

\section{USAGE EXAMPLE}

In this section, an explanation on the implementation of Autocarreq.Mereq by using a persona as is given as described below. Amin, an automotive engineer, wishes to evaluate the customer stakeholder's trust claim specification with AutocarreqMereq. He sits with Ahmad, a marketing manager, to analyze the needs he had previously captured. First of all, Amin takes part in an Ahmad meeting. Amin has to comprehend the aims of the project obviously during the conference in order to meet the needs of customers. Amin generates a set of textual specifications for autonomous cars as user scenarios at the conclusion of the conference. Amin inserts the demands as a user situation as shown in Figure 4 in the text editor (1) or he inserts the requirements by uploading the text* requirement specification (2). Then, he clicks the "Verify Trust" to capture the trust requirement (3). As shown in Figure 5.The phrases from the textual requirements are analyzed and matches using trust requirement autonomous car library to find an appropriate matching keywords with trust requirements (4). As shown in Figure 6 and Figure 7, after clicking "Verify Trust" then he shows the output such as the score of trust requirements (5) and Trustworthy percentage displayed (6) for the purpose analysing the earlier stage requirement given by Ahmad. If he is unhappy with the rest, the Amin can insert (7) and edit (8) the requirements based on the suggested trustworthy factor attributes (6). Amin confirms the trustworthy percentage of the capture requirements relation to earlier requirements provided by Ahmad. In order to allow Ahmad to have better understanding of the requirements components generated, Amin uses the MEReq tool map form model to perform the visualization of the mock up prototype for the associated analyzed trust requirements. Finally, Amin is able to confirm the percentage level of trust requirements in autonomous car with Ahmad for the earlier collected requirements. In summary, Amin uses the Autocarreq.Mereq tool to realize the autonomous trust requirements library that automatically captures the trust attributes user scenario provided by Ahmad earlier. He also uses the tool to manage and analyze the level of trust requirements before engineer can develop the autonomous cars. He then sits with Ahmad to verify and confirm the percentage of trust requirement before developing autonomous car by having the tool to generate the prototypes.

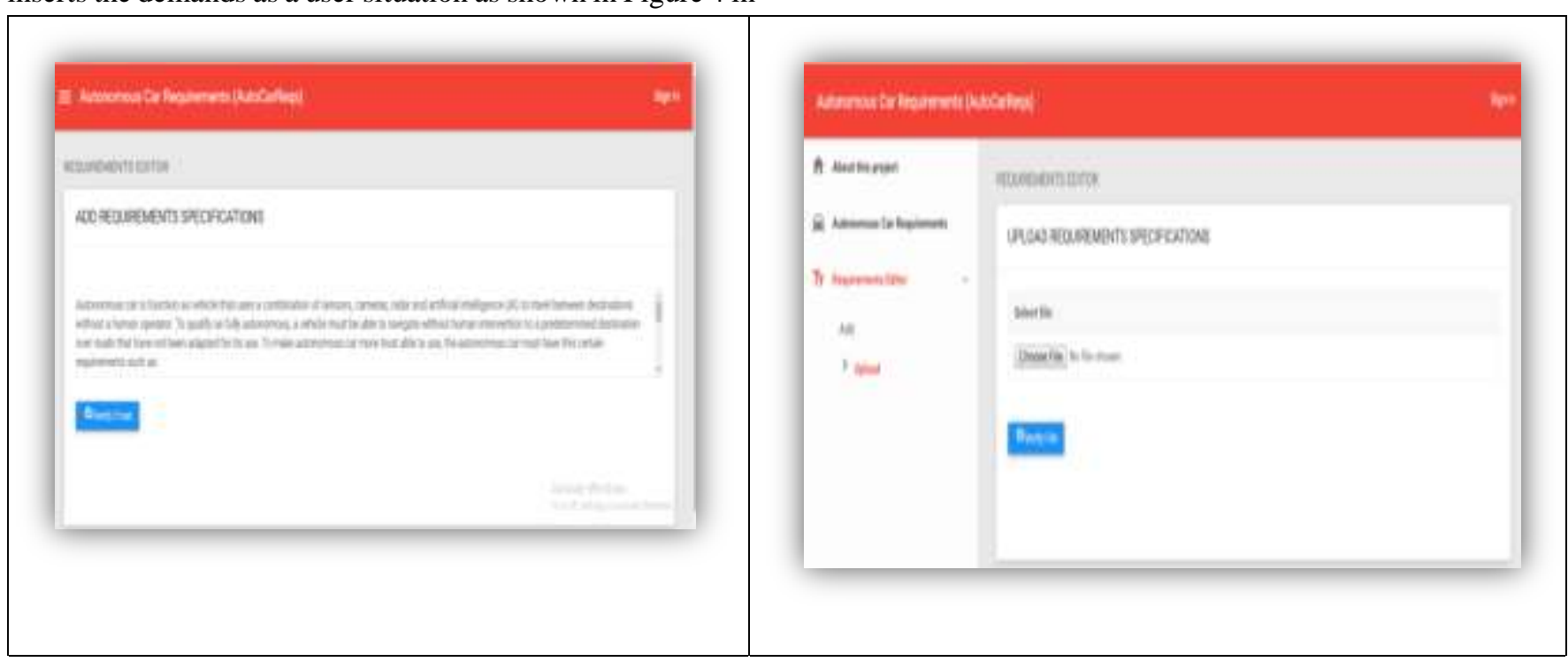

(1)

Figure 4: Insert and Upload Requirements Specification 


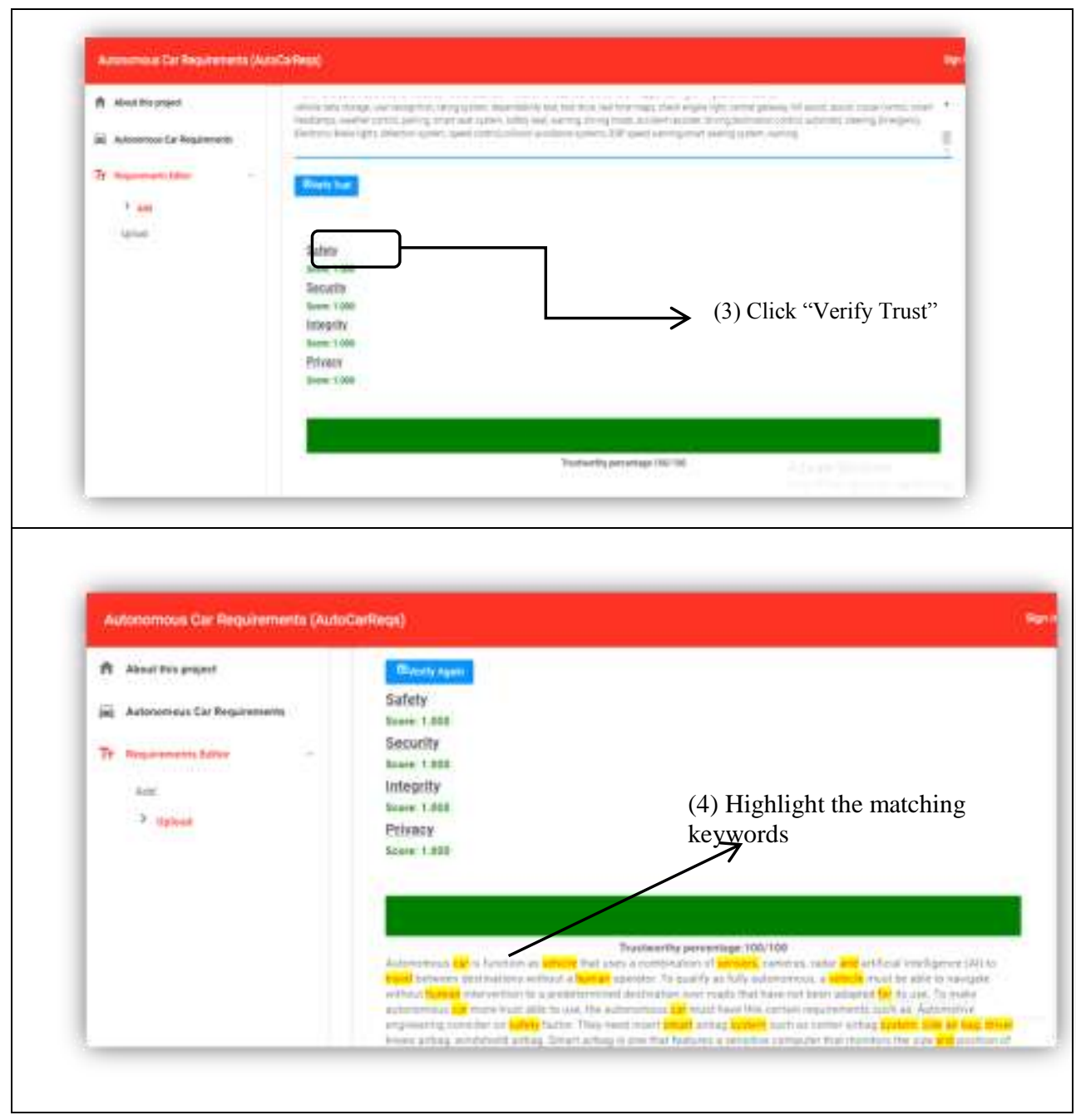

Figure 5: Verify Trust and Generated Matching Keywords

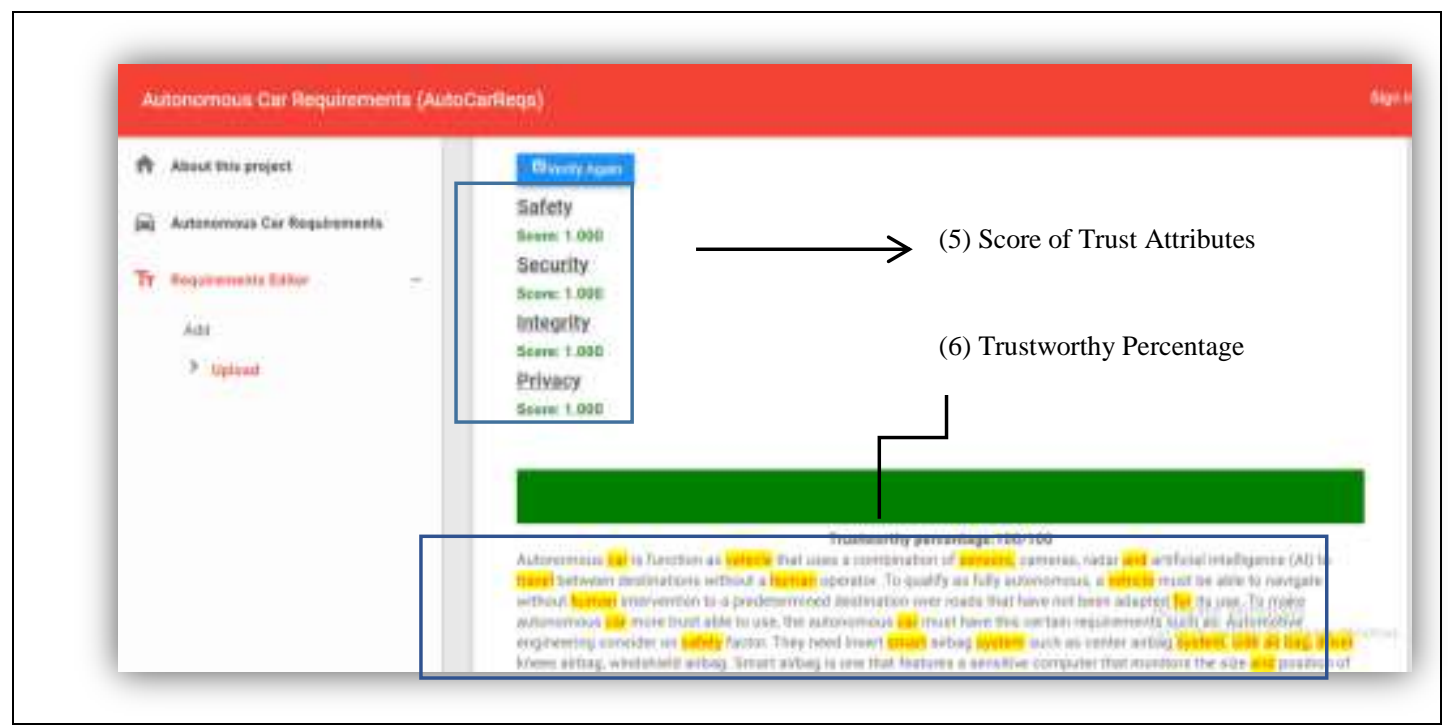

Figure 6: Trust Score

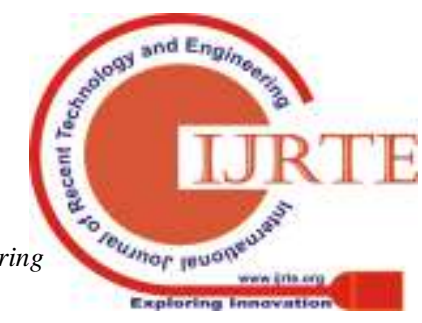




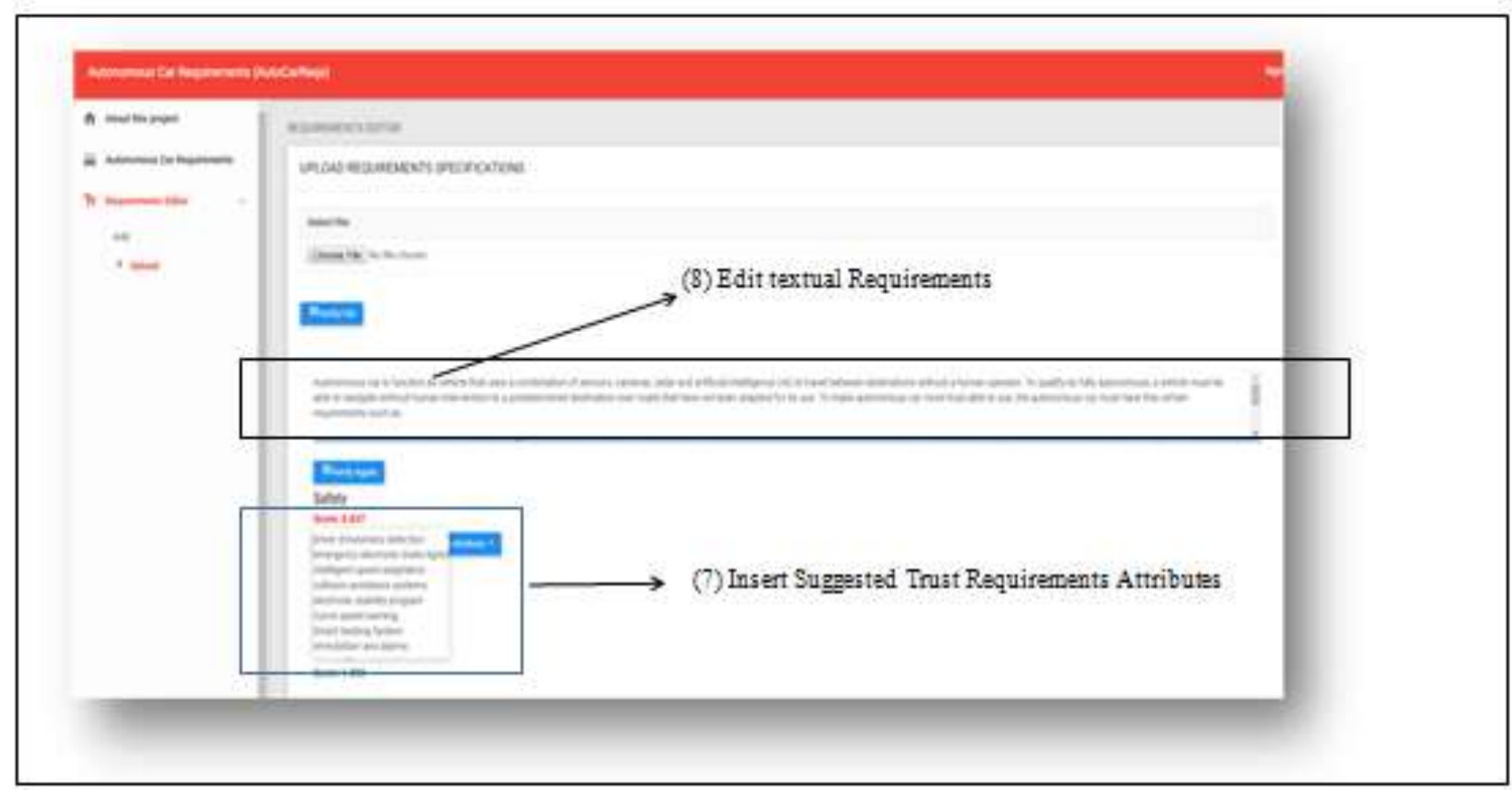

Figure 7: Insert and Edit Suggestion Trustworthy Attributes

\section{RESULT AND DISCUSSION}

40 final-year graduates, who study Software Engineering and have been registered in a Software Testing course have completed a usability assessment. This research aims to assess the usability of the automated tool to provide textual specifications. Two tasks were asked for the participation: one is the exploration of the tool with the sample requirements given (see Appendix A) and the other is the completion of a study questionnaire. After the assignment was completed, five issues concerning the usability of the tool had to be answered by the learners. The results of the tool usefulness, easy use, easy teaching and satisfaction surveys contained in Figure 9. 95\% of respondents found this tool to be helpful, $87 \%$ agreed that the tool is simple to use, $88 \%$ agreed that the tool was simple to learn and $86 \%$ agreed that the tool was satisfactory. Overall, the usability results show the usefulness, simplicity, easy-to-use, usability and usability of our prototype tool. They agree that the tool is easy to use, but want a better user interface and a easy user guide.

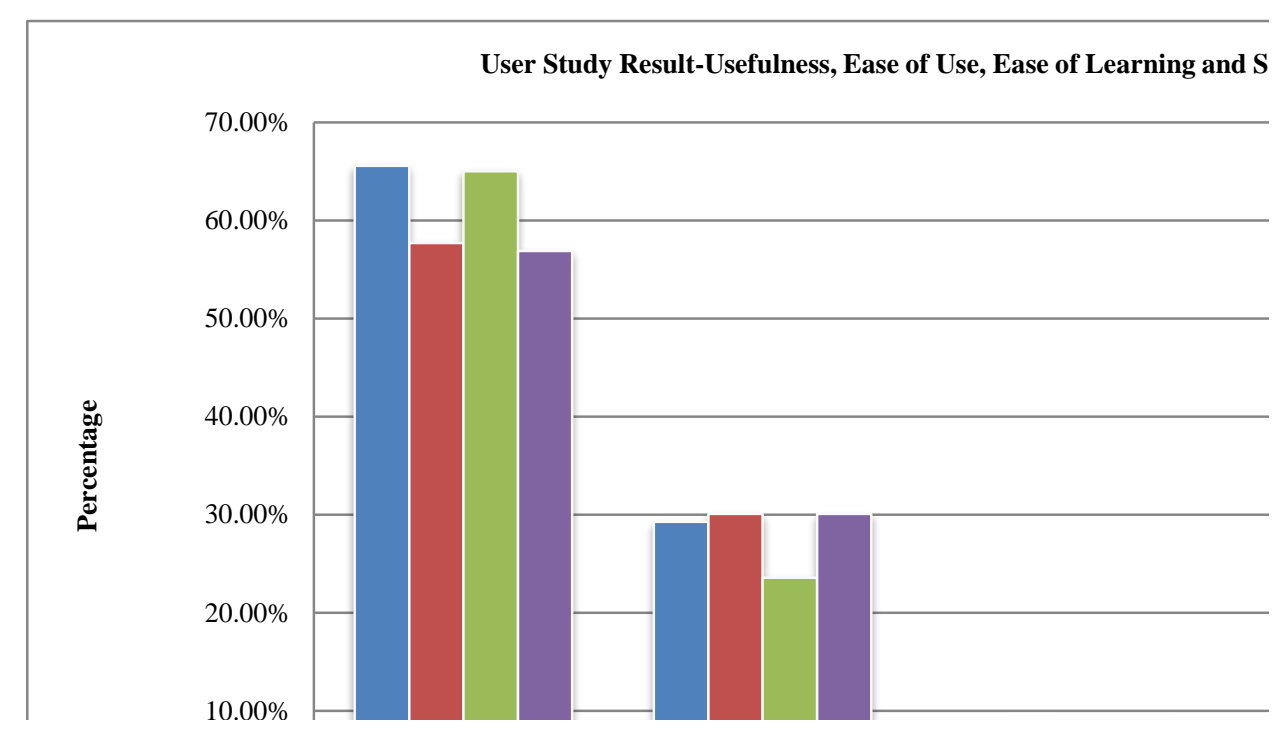




\section{CONCLUSIONS AND FUTURE WORKS}

Autocarreq.Mereq is a tool that assists the requirements elicitation process between automotive engineer and client-stakeholder. This tool automatically checks the level of trust by calculating the trust level using the keywords matching process by comparing the information in TReAC Library and from the textual requirements. The findings from evaluation indicated that our approach and tool support Autocarreq.Mereq is able to indicate that this automated tool is useful in helping the novice engineer without any background in automotive background in eliciting autonomous requirements. The result for this evaluation shows that generally, the participants agreed that the tool is helping them to capture correct trust requirements of autonomous. The participants also stated that the tool is helpful, convenient, time-consuming and particularly useful for novice users. Our future work is to improve this tool as a collaborative tool to enable better communication and discussion across geographic locations between clients and auto engineers.

\section{ACKNOWLEDGEMENT}

We would like to thank Universiti Teknikal Malaysia Melaka for its support. We also would like to thank our funder Zamalah UTeM, for sponsoring the author.

\section{REFERENCE}

1 NHSTSA, “Automated Vehicle for safety," 2016. [Online]. Available: https://www.nhtsa.gov/technology-innovation/automated -vehicles-safety.

2 G. M. Fitch, D. S. Bowman, and R. E. Llaneras, "Human Factors: The Journal of the Human Factors and Ergonomics Society," 2014.

3 M. Kamalrudin, J.Grundy and J.Hosking. "MEReq: A tool to capture and validate multi-lingual requirements." Frontiers in Artificial Intelligence and Applications, 265, 2014, pp.231-245.

4 V. Laporti, M. Borges, and V. Braganholo, "Athena: A collaborative approach to requirements elicitation. Computers in Industry", 60(6), 2009. pp.367-380.

5 J. Vijayan and G. Raju, "Requirements elicitation using paper prototype." Communications in Computer and Information Science, 117 CCIS, 2010. pp.30-37.

6 H. Hakimi, M.Kamalrudin, S.Sidek, and S. Akmal, "Trust Requirements Model for Developing Acceptable Autonomous Car" Journal of Electrical and Electronic Engineering. Vol. 6, No. 2, 2018, pp. 59-64. doi: $10.11648 /$ j.jeee.20180602.14 